\title{
Introduction for the First Issue
}

\author{
Stephen Billett
}

Published online: 9 January 2008

(C) Springer Science + Business Media B.V. 2007

This first edition of Vocations and Learning: Studies in vocational and professional education comprises contributions that indicate something of the journal's scope, focuses and purposes. Offered here are contributions from Canadian, British, Norwegian and German scholars that aim to understand further the nature of the occupations that individuals engage in and their learning for and through those occupations.

Tara Fenwick's paper discusses the experiences of women contract workers in the education and health sectors in Canada. Despite buoyant labour markets, it seems an increasing portion of workforces in advanced economies are becoming contract workers. This often requires individuals engaging in these forms of work to develop a range of capacities that go beyond those usually required for their occupational practice, yet are essential for their viability as workers. Fenwick reports this development often has to occur in relatively solitary circumstances of these workers' employment and engages them in negotiations with sometimes contradictory purposes, as identified in the paper. This work, the skills required, their development and these negotiations also have gendered qualities. Yet, these women's work is enacted within professional practices (i.e. education and health) where such negotiations may be easier for these women contract workers, than for those in other occupations. Although contract work is often aligned to involuntary contingent work and workers, a number of the participants in Fenwick's study reported electing to engage in this kind of work to secure more expansive and satisfying work roles. Indeed, the exercise of choice to take up this form of employment was selected by a number of these women because of disaffection with the restrictive and unfulfilling character of previous work roles. Some of these women were reported to possess

\footnotetext{
S. Billett $(\bowtie)$

School of Education and Professional Studies (Brisbane-Logan), Griffith University, Nathan 4111, Australia

e-mail: s.billett@griffith.edu.au
} 
qualities of creativity and adaptability that both fed that dissatisfaction and are now shaping their capacities to engage in these roles and negotiations. In this way, the paper illustrates instances of entanglements between the personal and the social factors that shape learning through and for work that features in much of Fenwick's theorising.

Phil Hodkinson, Gert Biesta and David James's paper on learning addresses this entanglement between social and individual from different perspectives. Their paper offers a view of learning that is inclusive of other accounts that variously emphasise individual and social perspectives. In particular, they hold that it is possible to combine the major elements of the situated and participatory views of learning which dominate current discussions. Within the context of learning for work, the authors engage with the notion of embodied construction as a cultural theory of learning that emphasises both social and individual contributions in this account. They do so with reference to Bourdieu's concepts of habitus and field, extended here to be inclusive of something shaped by practice, yet also needing to be experienced. In addition, the authors introduce the concept of scale to help assist understand how different accounts of social practices' contributions to shaping knowledge and learning can be understood in particular ways and levels of abstraction. In all, they offer a cultural theory of learning which positions individuals within and as part of that culture, not something separate from it and needing to be understood as being something separate from culture. This paper is particularly expansive and introduces readers to ways of viewing social practice in terms of scale, the individual as an embodiment of culture, and in this vein emphasises 'becoming' as a process of learning that arises through interactions and relations between the contributions of the learning culture and the position, habitus and capacities of the individuals who engage with these cultures. This conception is then used to respond to considerations of transfer as a process of 'becoming' that is enacted to a new learning situation. Also, in terms of learning efficacy, the authors suggest that questions about the improvement of learning are best considered in terms of relational cultural complexity, because individuals learn if needs and capacitors are so diverse as to obviate generalisable responses.

Monika Nerland's paper explores the conceptual basis for learning through work as participation in the epistemic cultures that comprises their occupational practice. Using the occupation of computer engineering as one that that embodies much of what is often presented in contemporary notions of knowledge work, and which has ambitions to be accepted as a major profession, this paper offers on account of the practices, codifications and objects associated with this occupational practice to elaborate conceptually their pedagogic or epistemic qualities. This elaboration draws extensively upon the work of Knorr Cetina, adapted here to appraise the specifics of actual occupational practice, as opposed to its previous application in less domain specific learning. The central case here is that the ways in which the occupational knowledge and its everyday practices are organised both reflects and constitutes its knowledge culture. In advancing its case, this paper refers to the way that this instance of professional knowledge is generated and distributed through global networks in ways quite distinct from other areas of professional practice. The knowledge required for the occupation rests more on constantly changing parameters and bases, rather than canonical principles and practices that have endured and been 
proven over time. Indeed, Nerland argues that this constant change means the work is often open-ended and ambiguous, rather than being directed towards fixed ends. Yet, regardless, and in partial contradiction, because of the architecture of this knowledge, and its use worldwide, there is need for standardisation and codifications for practice which is more analogous to professional practice as it is often currently understood. In all, the paper provides an account of the significance of what it refers to as 'epistemic tools and objects' to learning through for work. In doing so, the paper portrays the particular characteristics of knowledge culture of this occupation. The kinds of considerations provided by this paper seems salient to advancing our understanding of the genesis and manifestations of the knowledge required for work and also the ways in which that knowledge is engage with and learn by those who participate in the occupation. Consequently, it seems to augment and extend existing accounts of learning through and for occupational practice in so far as it suggests that occupations with their distinct geneses and manifestations, of themselves make particular contributions to the learning of those who participate in them.

Christian Harteis and Hans Gruber's paper also focuses on knowledge that is required for effective vocational practice, albeit from a different perspective that Nerland. Focussing more on individual capacities, they consider the role that intuition plays in experts' thinking and acting. They do this through examining the effectiveness of intuition in decision-making by novice and more experienced stockbrokers. The paper concludes that intuition plays a particularly vital role in work tasks when all of the knowledge required to arrive at effective decision-making is unknown or unknowable. The capacity to perform this kind of complex problem-solving in a domain of activity is often seen as the benchmark for expertise. The context for the investigation reported in this paper, is these kinds of circumstances in stock marketing work, where decisionmaking based on intuition is held to be more effective than rationally justified ones. Curiously, it was found that, overall, more novice stockbrokers performed as well as more experienced ones with their predictions, when rational decision-making was gauged. The exception was in a situation where the level of information was lower and the circumstance more difficult to predict. Here, the more experienced workers utilised intuitions in their more accurate predictions. Hence, it is proposed that in within a specific domain of occupational activity, intuition is an important capacity that constitutes an element of expert performance, particularly when some of the knowledge about the task is unknown. Importantly, this capacity seems to be one learnt through extensive experience within a domain of activities associated with paid work (i.e. stockbroking), suggesting it complements views about the development of domainspecific expertise from elsewhere. Moreover, it suggests that rather than this element of human performance being one that is broadly deployable that it is domain-specific. Indeed, its executive capacity seems to be through other domain-specific forms of knowledge upon which it relies and has been developed and through which it is enacted. Consequently, to develop such an attribute requires access to extensive episodes of engagements within a specific domain of activities. Much of what is proposed here might inform discussions about tacit knowledge, which seems well aligned to the definition of intuition adopted in this article. Again, it suggests that although individuals' capacity to manipulate knowledge (e.g. as in adapt) is likely to be present, that this alone is not sufficient. A repertoire of experiences is required to both develop and exercise intuition with effect. 
So, in some ways, the four contributions to this first issue are diverse in their focuses, orientations and contexts, as perhaps befits the broad field of vocational and professional learning. Yet, there are some common themes here. Negotiations of different kinds feature in Fenwick and Hodkinson et al.'s accounts of learning for work. In both, there is a consideration of how the contributions of the personal and the social are the bases for learning about, for and through work. Nerland's paper focuses on the epistemic qualities of particular kinds of work. However, like Fenwick and Hodkinson's paper, Nerland also prefers to the ways in which workers in this occupation exercise flexibility and make particular choices in how they engage with the knowledge culture that comprises that work. Indeed, like the Fenwick paper, she points to the need for these workers to exercise reflexivity and self-management. Harteis and Gruber's paper examines what has come to be learnt through such process: intuition and its role in thinking and acting at work. Such capacities arise from and contribute to subsequent learning through processes of the kind that the other three papers propose. 